\title{
BOREL SUBGROUPS ADAPTED TO NILPOTENT ELEMENTS OF STANDARD LEVI TYPE
}

\author{
LUCAS FRESSE
}

\begin{abstract}
Let a reductive algebraic group over an algebraically closed field of good characteristic be given. Attached to a nilpotent element of its Lie algebra, we consider a family of algebraic varieties, which incorporates classical objects such as Springer fiber, Spaltenstein varieties, and Hessenberg varieties. When the nilpotent element is of standard Levi type, we show that the varieties of this family admit affine pavings that can be obtained by intersecting with the Schubert cells corresponding to a suitable Borel subgroup.
\end{abstract}

\section{INTRODUCTION}

Let $G$ be a connected reductive linear algebraic group over $\mathbb{K}$, an algebraically closed field. The characteristic of $\mathbb{K}$ is assumed to be good for $G$. We denote by $\mathfrak{g}$ the Lie algebra of $G$. We are interested in nilpotent elements $e \in \mathfrak{g}$ and, especially, we consider the following object.

Definition 1. Given a nilpotent element $e \in \mathfrak{g}$, a parabolic subgroup $P \subset G$, and a subspace $V \subset \mathfrak{g}$ stabilized by the adjoint action of $P$, we define

$$
\mathcal{P}_{e, V}=\left\{g P \in G / P: g^{-1} \cdot e \in V\right\} .
$$

Hereafter, $(g, x) \mapsto g \cdot x$ denotes the adjoint action $G \times \mathfrak{g} \rightarrow \mathfrak{g}$. The set $\mathcal{P}_{e, V}$ is then a closed (possibly empty) subvariety of the partial flag variety $G / P$. As noted in the following example, some classical objects occurring in representation theory fit in Definition 1

Example 1. (a) Let $P=B$ be a Borel subgroup of $G$ and let $V=\mathfrak{n}$ be the nilradical of the corresponding Borel subalgebra of $\mathfrak{g}$. Then, $\mathcal{P}_{e, V}$ coincides with the fiber over $e$ of the map $G \times{ }^{B} \mathfrak{n} \rightarrow G \cdot \mathfrak{n}$. This map is the Springer resolution of the nilpotent cone of $\mathfrak{g}$. The variety $\mathcal{B}_{e}:=\mathcal{P}_{e, V}$ is called a Springer fiber.

(b) Let $P \subset G$ be a parabolic subgroup. If $V=\mathfrak{p}$ is the corresponding parabolic subalgebra of $\mathfrak{g}$, then the variety $\mathcal{P}_{e, V}=\mathcal{P}_{e, \mathfrak{p}}$ is called a Steinberg variety, studied in [20]. If $V=\mathfrak{n}_{P}$ is the nilradical of $\mathfrak{p}$, then the variety $\mathcal{P}_{e, V}=\mathcal{P}_{e, \mathfrak{n}_{P}}$ is called a Spaltenstein variety. It coincides with the fiber over $e$ of the natural map $G \times{ }^{P} \mathfrak{n}_{P} \rightarrow$ $G \cdot \mathfrak{n}_{P}$. The set $G \cdot \mathfrak{n}_{P}$ is the closure of the Richardson nilpotent orbit associated to $P$ and this map is a quasi-resolution (i.e., proper, surjective, generically finite to one, see [2, 3]), which generalizes the Springer resolution. If $P=B$ is a Borel subgroup, then both varieties $\mathcal{P}_{e, \mathfrak{p}}$ and $\mathcal{P}_{e, \mathfrak{n}_{P}}$ coincide with the Springer fiber $\mathcal{B}_{e}$.

Received by the editors February 10, 2014 and, in revised form, July 15, 2014.

2010 Mathematics Subject Classification. Primary 17B08, 20G07, 14M15.

Key words and phrases. Nilpotent orbits, standard Levi type, partial flag varieties, Springer fibers, affine pavings.

This work was supported in part by the ANR project NilpOrbRT (ANR-12-PDOC-0031). 
(c) Assume $\mathbb{K}$ of characteristic zero. Let $e \in \mathfrak{g}$ be a nilpotent element. By the Jacobson-Morozov lemma, we can embed $e$ in a triple $\{e, h, f\} \subset \mathfrak{g}$ such that $[h, e]=2 e,[h, f]=-2 f$, and $[e, f]=h$. The semisimple element $h$ induces a grading $\mathfrak{g}=\bigoplus_{i \in \mathbb{Z}} \mathfrak{g}_{i}$ where $\mathfrak{g}_{i}=\{x \in \mathfrak{g}:[h, x]=i x\}$. Write $\mathfrak{g}_{\geq j}=\bigoplus_{i>j} \mathfrak{g}_{i}$. Then $\mathfrak{p}:=\mathfrak{g}_{\geq 0}$ is a parabolic subalgebra of $\mathfrak{g}$, corresponding to a parabolic subgroup $P \subset G$, and $V:=\mathfrak{g}_{\geq 2}$ is a $P$-stable subspace. We have $e \in \mathfrak{g}_{2}$, in fact it turns out that $G \cdot \mathfrak{g}_{\geq 2}=\overline{G \cdot e}$, and the natural map $G \times{ }^{P} \mathfrak{g}_{\geq 2} \rightarrow G \cdot \mathfrak{g}_{\geq 2}$ is a resolution of the nilpotent orbit closure $\overline{G \cdot e}$, called Dynkin resolution (see [14]). The fiber over $e^{\prime} \in \overline{G \cdot e}$ of this resolution coincides with the variety $\mathcal{P}_{e^{\prime}, V}$.

(d) Let $P=B$ be a Borel subgroup with Lie algebra $\mathfrak{b} \subset \mathfrak{g}$ and let $V \subset \mathfrak{g}$ be a $B$-stable subspace which contains $\mathfrak{b}$. Then $V$ is called a Hessenberg space and the variety $\mathcal{P}_{e, V}$ is called a Hessenberg variety (see [8]). In the case where $V=\mathfrak{b}$, we retrieve the Springer fiber $\mathcal{P}_{e, V}=\mathcal{B}_{e}$.

In this paper, we mainly focus on nilpotent elements of standard Levi type. By virtue of Bala-Carter theory, for every nilpotent element $e \in \mathfrak{g}$, there is a minimal Levi subalgebra $\mathfrak{g}_{0} \subset \mathfrak{g}$ (unique up to conjugation) containing $e$. Then, we say that $e$ is of standard Levi type if it is a regular nilpotent element of $\mathfrak{g}_{0}$. If $G=G L_{n}(\mathbb{K})$, then every nilpotent element of $\mathfrak{g}=\mathfrak{g l}_{n}(\mathbb{K})$ is of standard Levi type. In general, the nilpotent elements of standard Levi type do not exhaust all the nilpotent elements. In Section 2, we review the basic properties of the nilpotent elements of this type. In Section [5, we show the following characterization:

Proposition 1. Given a nilpotent element $e \in \mathfrak{g}$, the following conditions are equivalent:

(i) $e$ is of standard Levi type;

(ii) the group $N_{G}(e):=\{g \in G: g \cdot e \in \mathbb{K} e\}$ contains a regular, rank-one subtorus $H=\left\{\eta(t): t \in \mathbb{K}^{*}\right\} \subset G$.

By regular subtorus, we mean a torus that contains regular semisimple elements of $G$, or equivalently a torus that admits a finite set of fixed points for its action on any partial flag variety $G / P$. If $H$ is as in Proposition 1, then it also acts on any subvariety of the form $\mathcal{P}_{e, V} \subset G / P$ with a finite number of fixed points and this action stabilizes every irreducible component of $\mathcal{P}_{e, V}$. Invoking Białynicki-Birula's theorem (see [1, §4]), we have the following consequence of Proposition 1

Corollary 1. Assume that the nilpotent element $e \in \mathfrak{g}$ is of standard Levi type. Let $\mathcal{P}_{e, V}$ be a variety like in Definition 1 and let $X \subset \mathcal{P}_{e, V}$ be a smooth irreducible component. Then, $X$ admits an affine paving (i.e., there is a decomposition $X=$ $\bigsqcup_{i=1}^{k} X_{i}$ such that $X_{1} \sqcup \ldots \sqcup X_{i}$ is closed for all $i \in\{1, \ldots, k\}$ and $X_{i}$ is isomorphic to an affine space for all $i$ ).

Note that the variety $\mathcal{P}_{e, V}$ may not be irreducible or smooth, which prevents us from directly applying Białynicki-Birula's theorem to obtain Corollary 1 for the whole variety $\mathcal{P}_{e, V}$. Actually our main purpose is to strengthen the conclusion of Corollary 1 and to extend it to the whole variety $\mathcal{P}_{e, V}$. The main result of the paper is the following theorem.

Theorem 1. Assume that the nilpotent element $e \in \mathfrak{g}$ is of standard Levi type. Then, there is a Borel subgroup $B \subset G$ satisfying the following properties: 
(a) For every parabolic subgroup $P \subset G$ and every $P$-stable subspace $V \subset \mathfrak{g}$, the intersection of the variety $\mathcal{P}_{e, V}$ with any $B$-orbit of the partial flag variety $G / P$ (if nonempty) is isomorphic to an affine space.

(b) Moreover, for every smooth irreducible component $X \subset \mathcal{P}_{e, V}$, the intersection of $X$ with any $B$-orbit of $G / P$ is isomorphic to an affine space.

In particular, Theorem 1 establishes the existence of an affine paving for the varieties $\mathcal{P}_{e, V}$ associated to the nilpotent elements of standard Levi type. The existence of an affine paving guarantees good (co)homological properties (we refer to [7, $\S 1]$ and [11, $\S \S 11-12]$ for an overview of the notion of affine pavings) and is especially desirable for varieties arising as fibers of resolutions (see [12]).

It is already known (cf. [7, 17]) that a Springer fiber $\mathcal{B}_{e}$ always admits an affine paving whenever $e$ is a nilpotent element (not necessarily of standard Levi type) with no term of type $E_{7}$ or $E_{8}$ in the minimal Levi subalgebra that contains it (and the result is speculated to be true also without this restriction). Constructions of affine pavings for Springer fibers are also discussed in 13, 18, 22. The existence of affine pavings for type $A$ Steinberg and Spaltenstein varieties is established in [16] and [4]. In [16], the paving of the Steinberg variety is obtained, as in Theorem 1], by intersecting with certain $B$-orbits. The existence of affine pavings for Hessenberg varieties associated to nilpotent elements of standard Levi type is shown (when the base field is of characteristic zero) in 21. (type $A$ case) and 15. (semisimple case), where the affine paving is also obtained by intersecting with certain $B$-orbits of the flag variety. Actually, the results in [15, 21] are more general since they concern Hessenberg varieties associated to (not necessarily nilpotent) elements of the form $e=e_{s}+e_{n}$ with $e_{s}$ semisimple, $e_{n}$ nilpotent of standard Levi type, $\left[e_{s}, e_{n}\right]=0$.

The main novelty of Theorem [1 with respect to the existing literature is that the affine paving obtained is explicit and of a very special form, the construction is valid once the characteristic of the base field $\mathbb{K}$ is good for $G$, and the construction is canonical in the sense that the same Borel subgroup $B$ allows to obtain pavings of all the varieties $\mathcal{P}_{e, V}$ (and of all their smooth components) for all choices of $P$ and $V$. By combining Theorem 1 and Example 1(c), we also get a positive answer to [12, Question 4.19] for nilpotent elements of standard Levi type.

The paper is organized as follows. In Section 2, we recall basic facts on nilpotent elements of standard Levi type (the definition, their relation to Bala-Carter theory, and their classification). The main tool used in the paper is the notion of cocharacter associated to a nilpotent element, which is recalled in Section 3 . This notion plays a crucial role for the study of nilpotent elements in good characteristic. The proof of Theorem 1 relies on general constructions done in Section 4, inspired by ideas developed in [7] and [11. In Section [5] we give the proofs of Proposition 1 and Theorem 1 . The group $B$ fulfilling the conditions of Theorem 1 is explicit and its construction is explained in detail in Sections 45 (see Theorem 4, which is a more precise version of Theorem 1). Some concrete examples are presented in Section 6 .

\section{Nilpotent Elements of Standard LeVi type}

The beginning of this section reviews some basic notions related to nilpotent elements and nilpotent orbits. The references are [5] and [11].

Recall that an element $e \in \mathfrak{g}$ is nilpotent if it belongs to $[\mathfrak{g}, \mathfrak{g}]$ and satisfies that ad $e: \mathfrak{g} \rightarrow \mathfrak{g}$ is a nilpotent endomorphism. The set $\mathcal{N} \subset \mathfrak{g}$ of nilpotent elements is 
an irreducible, closed subvariety, consisting of finitely many adjoint $G$-orbits called nilpotent orbits and, when the characteristic of the base field $\mathbb{K}$ is good (as supposed here), these orbits can be classified in terms of Bala-Carter theory, as described below. The regular nilpotent elements, i.e., those satisfying

$$
\operatorname{dim} Z_{G}(e)=\operatorname{rank} G, \quad \text { where } Z_{G}(e)=\{g \in G: g \cdot e=e\},
$$

form a single open nilpotent orbit.

A nilpotent element $e$ is distinguished if every torus contained in $Z_{G}(e)$ is contained in the center of $G$. The regular nilpotent elements are distinguished, and, if $G=G L_{n}(\mathbb{K})$, then the converse is also true.

If $S \subset G$ is a torus, then its centralizer $Z_{G}(S) \subset G$ is a connected reductive group of Lie algebra $\mathfrak{z}_{\mathfrak{g}}(S):=\{x \in \mathfrak{g}: s \cdot x=x \forall s \in S\}$. We call $Z_{G}(S)$ a Levi subgroup (and $\mathfrak{z}_{\mathfrak{g}}(S)$ a Levi subalgebra) as it can be realized as a Levi factor of some parabolic subgroup and, conversely, a Levi factor of a parabolic subgroup is the centralizer $Z_{G}(S)$ of some torus $S \subset G$.

Proposition 2 (Bala-Carter). Let $e \in \mathfrak{g}$ be a nilpotent element.

(a) There exists a Levi subgroup $L \subset G$ such that e is a distinguished element of the Lie algebra $\operatorname{Lie}(L)$.

(b) More precisely, the Levi subgroups satisfying the property in (a) are exactly those of the form $Z_{G}(S)$ where $S$ is a maximal torus of $Z_{G}(e)$.

(c) In particular, any two Levi subgroups satisfying (a) are conjugated under an element of the connected group $Z_{G}(e)^{0}$.

A nilpotent element $e \in \mathfrak{g}$ is Richardson if there is a parabolic subgroup $P \subset G$ such that $P \cdot e$ is an open subset of the nilradical of the Lie algebra Lie $(P)$. Then, $G \cdot e$ is a Richardson nilpotent orbit (all of its elements are Richardson). For example, if $e$ is regular, then $e$ is Richardson, corresponding to $P=B$, where $B \subset G$ is the unique Borel subgroup with $e \in \operatorname{Lie}(B)$. At the other extreme, $e=0$ is also Richardson, corresponding to $P=G$.

A parabolic subgroup $P$ of the derived group $G^{\prime} \subset G$ is called distinguished if $\operatorname{dim} P / U_{P}=\operatorname{dim} U_{P} / U_{P}^{\prime}$, where $U_{P}^{\prime} \subset U_{P} \subset P$ denote the unipotent radical of $P$ and its derived group. When the characteristic of $\mathbb{K}$ is good, the notions of distinguished parabolic subgroups and distinguished nilpotent elements match well.

Theorem 2 (Bala-Carter). A nilpotent element $e \in \mathfrak{g}$ is distinguished if and only if it is a Richardson nilpotent element corresponding to a distinguished parabolic subgroup $P \subset G^{\prime}$. Moreover, the map

$$
(L, P) \mapsto G \cdot e,
$$

which maps the pair formed by a Levi subgroup $L \subset G$ and a distinguished parabolic subgroup $P \subset L^{\prime}$ to the $G$-orbit of a Richardson element corresponding to $P$, induces a bijection between the set of $G$-orbits of such pairs $(L, P)$ and the set of nilpotent orbits of $\mathfrak{g}$.

In this paper, we focus on the following family of nilpotent elements.

Definition 2. Let $e \in \mathfrak{g}$ be a nilpotent element. We say that $e$ is of standard Levi type if there is a Levi subgroup $L$ such that $e$ is a regular nilpotent element of $\operatorname{Lie}(L)$. Then, we say that $G \cdot e$ is a nilpotent orbit of standard Levi type. 
In terms of Bala-Carter theory, the nilpotent orbits of standard Levi type are those corresponding to ( $G$-orbits of) pairs of the form $\left(L, B_{L}\right)$ where $L \subset G$ is a Levi subgroup and $B_{L}$ is a Borel subgroup of $L^{\prime}$. The regular nilpotent orbit is of standard Levi type (corresponding to the pair $(G, B)$ where $B \subset G^{\prime}$ is a Borel subgroup). At the other extreme, $e=0$ is of standard Levi type (corresponding to the pair $\left(T,\left\{1_{G}\right\}\right)$ where $T \subset G$ is a maximal torus).

Example 2. Suppose that the Lie algebra $\mathfrak{g}$ is simple.

(a) In type $A_{n-1}$, that is, if $\mathfrak{g}=\mathfrak{s l}_{n}(\mathbb{K})$, then every nilpotent element $e$ is of standard Levi type. Indeed, up to conjugation $e$ is characterized by the sizes of its Jordan blocks $\left(\pi_{1} \geq \ldots \geq \pi_{k}\right)$ which form a partition of $n$, namely (up to conjugation) $e$ is a standard Jordan matrix

$$
\left(\begin{array}{ccc}
e_{\pi_{1}} & & 0 \\
& \ddots & \\
0 & & e_{\pi_{k}}
\end{array}\right)
$$

where $e_{\pi_{i}}$ is a matrix whose coefficients are all zero except those on the upper subdiagonal, which are equal to 1 . Thus, $e$ is regular in the Levi subalgebra $\mathfrak{l} \subset$ $\mathfrak{s l}_{n}(\mathbb{K})$ formed by blockwise diagonal matrices with blocks of sizes $\pi_{1}, \ldots, \pi_{k}$ along the diagonal.

(b) In the other classical cases $B_{n}, C_{n}, D_{n}$, the nilpotent orbits are not all of standard Levi type. We refer to [10, Appendix 3] or Section 6.2 where the nilpotent orbits of standard Levi type are described in terms of admissible partitions.

(c) The following table indicates the number of nilpotent orbits of standard Levi type in the exceptional cases (see [6, §8.4]).

\begin{tabular}{||c|c|c||}
\hline type & $\begin{array}{l}\text { number of nilpotent } \\
\text { orbits }\end{array}$ & $\begin{array}{l}\text { number of orbits of } \\
\text { standard Levi type }\end{array}$ \\
\hline$G_{2}$ & 4 & 3 \\
\hline$F_{4}$ & 16 & 12 \\
\hline$E_{6}$ & 21 & 17 \\
\hline$E_{7}$ & 45 & 32 \\
\hline$E_{8}$ & 70 & 41 \\
\hline
\end{tabular}

(d) The trivial, minimal, and regular nilpotent orbits of $\mathfrak{g}$ are always of standard Levi type. The subregular nilpotent orbit of $\mathfrak{g}$ is of standard Levi type if and only if $\mathfrak{g}$ is of type $A_{n}, B_{n}, D_{n}$, or $C_{2}$.

Remark 1. (a) Let $P \subset G$ be a parabolic subgroup. We denote by $\mathcal{O}_{P} \subset \mathfrak{g}$ the Richardson nilpotent orbit corresponding to $P$ (defined above). Let $L \subset P$ be a Levi factor and let $e \in \operatorname{Lie}(L)$ be a regular nilpotent element, then $\mathcal{O}_{P}^{\prime}:=G \cdot e$ is a nilpotent orbit of standard Levi type and it is independent of the choice of the Levi factor $L$ and of the element $e$. Every Richardson nilpotent orbit (resp. every nilpotent orbit of standard Levi type) is of the form $\mathcal{O}_{P}$ (resp. $\mathcal{O}_{P}^{\prime}$ ) for some parabolic subgroup $P \subset G$. However, different parabolic subgroups (even if not conjugate) can give rise to the same orbits.

(b) The map $\mathcal{O}_{P}^{\prime} \mapsto \mathcal{O}_{P}$ is, nevertheless, well defined, surjective, but in general not injective: the nilpotent orbits of standard Levi type are in general more numerous than the Richardson nilpotent orbits (see [10, §2.5]).

(c) Actually, it is known from Spaltenstein [18, §III] that there is a duality map $d$ on the set of nilpotent orbits, satisfying (in fact, almost characterized by) the 
properties $d \circ d \circ d=d$ (i.e., $d$ is an involution on its image) and $d\left(\mathcal{O}_{P}^{\prime}\right)=\mathcal{O}_{P}$ for every parabolic subgroup $P \subset G$. If $\mathfrak{g}=\mathfrak{s l}_{n}(\mathbb{K})$, then the duality $d$ is the bijection $\mathcal{O}_{\pi} \mapsto \mathcal{O}_{\pi^{*}}$, which maps the nilpotent orbit $\mathcal{O}_{\pi}$ corresponding to a partition $\pi=$ $\left(\pi_{1} \geq \ldots \geq \pi_{k}\right)$ to the nilpotent orbit $\mathcal{O}_{\pi^{*}}$ corresponding to the dual partition $\pi^{*}=\left(\pi_{1}^{*} \geq \ldots \geq \pi_{\pi_{1}}^{*}\right)$ defined by $\pi_{j}^{*}=\left|\left\{i=1, \ldots, k: \pi_{i} \geq j\right\}\right|$.

\section{Cocharacters associated to nilpotent Elements}

A cocharacter is a morphism of algebraic groups $\tau: \mathbb{K}^{*} \rightarrow G$. In particular, a cocharacter defines a $\mathbb{Z}$-grading of the Lie algebra

$$
\mathfrak{g}=\bigoplus_{i \in \mathbb{Z}} \mathfrak{g}(i ; \tau)
$$

where

$$
\mathfrak{g}(i ; \tau)=\left\{x \in \mathfrak{g}: \tau(t) \cdot x=t^{i} x\right\} .
$$

A cocharacter also defines a parabolic subgroup

$$
Q(\tau)=\left\{g \in G: \lim _{t \rightarrow 0} \tau(t) g \tau(t)^{-1} \text { exists }\right\}
$$

and a Levi decomposition $Q(\tau)=L(\tau) U(\tau)$, where

$$
\begin{aligned}
U(\tau) & =\left\{g \in G: \lim _{t \rightarrow 0} \tau(t) g \tau(t)^{-1}=1_{G}\right\} \\
\text { and } \quad L(\tau) & =Z_{G}(\tau):=\left\{g \in G: \tau(t) g=g \tau(t) \quad \forall t \in \mathbb{K}^{*}\right\} .
\end{aligned}
$$

Moreover, the Lie algebras of $Q(\tau), U(\tau)$, and $L(\tau)$ are, respectively,

$$
\operatorname{Lie}(Q(\tau))=\bigoplus_{i \geq 0} \mathfrak{g}(i ; \tau), \quad \operatorname{Lie}(U(\tau))=\bigoplus_{i \geq 1} \mathfrak{g}(i ; \tau), \quad \operatorname{Lie}(L(\tau))=\mathfrak{g}(0 ; \tau)
$$

The following notion plays a key role in what follows (see [11, §5.3]).

Definition 3. Let $e \in \mathfrak{g}$ be a nilpotent element. A cocharacter $\tau: \mathbb{K}^{*} \rightarrow G$ is said to be associated to $e$ if the following conditions are satisfied:

(a) $e \in \mathfrak{g}(2 ; \tau)$;

(b) there is a Levi subgroup $L \subset G$ such that $e$ is distinguished in $\operatorname{Lie}(L)$ and $\tau\left(\mathbb{K}^{*}\right) \subset L^{\prime}$.

We have (see [11, Lemma 5.3]):

Proposition 3. Let $e \in \mathfrak{g}$ be a nilpotent element. Then, there exists a cocharacter associated to e. Two cocharacters associated to e are conjugate under $Z_{G}(e)^{0}$.

We also point out the following simple consequence of Definition 3(a):

Lemma 1. Let $e \in \mathfrak{g}$ be nilpotent and let $\tau: \mathbb{K}^{*} \rightarrow G$ be a cocharacter associated to $e$. Then, we have

$$
N_{G}(e)=\tau\left(\mathbb{K}^{*}\right) Z_{G}(e) .
$$

Proof. By Definition 3 (a) and the fact that $\mathbb{K}$ is algebraically closed, we have

$$
\tau\left(\mathbb{K}^{*}\right) \cdot e=\left\{t^{2} e: t \in \mathbb{K}^{*}\right\}=\mathbb{K}^{*} e .
$$

Then, for every $g \in N_{G}(e)$, we can find $t \in \mathbb{K}^{*}$ such that $g \cdot e=\tau(t) \cdot e$, i.e., $g \in \tau(t) Z_{G}(e)$.

The next statement underlines the properties of the parabolic subgroup $Q(\tau)$ for $\tau$ as in Definition 3 (see [11, Proposition 5.9]). 
Proposition 4. Let $e \in \mathfrak{g}$ be a nilpotent element and let $\tau: \mathbb{K}^{*} \rightarrow G$ be a cocharacter associated to $e$. The parabolic subgroup $Q(\tau)$ corresponding to $\tau$ satisfies the following properties:

(a) $\overline{Q(\tau) \cdot e}=\bigoplus_{i \geq 2} \mathfrak{g}(i ; \tau)$;

(b) $Z_{G}(e) \subset Q(\tau)$;

(c) $Q(\tau)$ is independent of $\tau$, that is, if $\tau^{\prime}: \mathbb{K}^{*} \rightarrow G$ is another cocharacter associated to e, then $Q\left(\tau^{\prime}\right)=Q(\tau)$.

We conclude this section with the characterizations of the cocharacters associated to the distinguished and the regular nilpotent elements.

Lemma 2. Let $e \in \mathfrak{g}$ be a nilpotent element and let $\tau: \mathbb{K}^{*} \rightarrow G$ be a cocharacter associated to $e$. If $e$ is a distinguished nilpotent element, then the grading induced by $\tau$ is even, that is, we have $\mathfrak{g}(i ; \tau)=0$ whenever $i$ is odd.

Proof. It follows from the proof of [11, Lemma 5.3(a)] that there exists a cocharacter associated to $e$, which induces an even grading of $\mathfrak{g}$. We deduce from Proposition 3 that every cocharacter associated to $e$ fulfills this property.

Lemma 3. Let $e \in \mathfrak{g}$ be a distinguished nilpotent element and let $\tau: \mathbb{K}^{*} \rightarrow G$ be a cocharacter associated to $e$. The following conditions are equivalent:

(i) $e$ is a regular nilpotent element;

(ii) $\tau$ is a regular cocharacter, i.e., $Z_{G}(\tau)$ is a (maximal) torus of $G$.

Proof. We write $Q=Q(\tau), \mathfrak{q}=\bigoplus_{i \geq 0} \mathfrak{g}(i ; \tau), \mathfrak{l}=\mathfrak{g}(0 ; \tau)$, and $\mathfrak{m}=\bigoplus_{i \geq 2} \mathfrak{g}(i ; \tau)$. The assumption and Lemma 2 guarantee that $\mathfrak{q}=\mathfrak{l} \oplus \mathfrak{m}$. Using Proposition 4 (a) and (b), we obtain

$$
\operatorname{dim} Z_{G}(\tau)=\operatorname{dim} \mathfrak{l}=\operatorname{dim} Q-\operatorname{dim} Q \cdot e=\operatorname{dim} Z_{Q}(e)=\operatorname{dim} Z_{G}(e) .
$$

It follows:

$$
\operatorname{dim} Z_{G}(\tau)=\operatorname{rank} G \Leftrightarrow \operatorname{dim} Z_{G}(e)=\operatorname{rank} G .
$$

Whence the equivalence between (i) and (ii).

\section{Borel SUbGroups ADAPTED TO NILPOTENT ELEMENTS}

In this section, we suppose that $e \in \mathfrak{g}$ is a nilpotent element. We do preliminary constructions that involve a cocharacter $\tau$ associated to $e$, the corresponding parabolic subgroup $Q(\tau)$, and a Borel subgroup adapted to these data. In the next section, we will specialize to the case where $e$ is of standard Levi type.

Throughout this section, we fix the following notation:

- Let $S_{0}$ be a maximal torus of $Z_{G}(e)$;

- Let $G_{0}=Z_{G}\left(S_{0}\right)$, so that $e$ is a distinguished nilpotent element of $\mathfrak{g}_{0}:=$ $\operatorname{Lie}\left(G_{0}\right)$ (see Proposition 2(b));

- Let $\tau: \mathbb{K}^{*} \rightarrow G$ be a cocharacter associated to $e$ such that $\tau\left(\mathbb{K}^{*}\right) \subset G_{0}^{\prime}$ (it exists by virtue of Proposition 2(c) and Proposition 3) and let $Q(\tau) \subset G$ be the corresponding parabolic subgroup;

- Note that $S_{0}$ and $\tau$ generate a torus $S_{0} \tau\left(\mathbb{K}^{*}\right)$, contained in $G_{0}$ and $Q(\tau)$. 
Definition 4. With the notation as above, we say that a Borel subgroup $B \subset G$ is adapted to the pair $\left(S_{0}, \tau\right)$ if it satisfies the following conditions:

(a) $S_{0} \tau\left(\mathbb{K}^{*}\right) \subset B \subset Q(\tau)$;

(b) $B \cap L(\tau)$ is contained in a parabolic subgroup of $G$ admitting $G_{0}$ as Levi factor.

Lemma 4. Borel subgroups adapted to $\left(S_{0}, \tau\right)$ in the sense of Definition 4 always exist.

Proof. Take $Q_{0} \subset G$ a parabolic subgroup admitting $G_{0}$ as Levi factor. Then $Q_{0} \cap L(\tau)$ is a parabolic subgroup of $L(\tau)$ and there is a Borel subgroup $B_{1}$ of $L(\tau)$ such that $S_{0} \tau\left(\mathbb{K}^{*}\right) \subset B_{1} \subset Q_{0} \cap L(\tau)$. Set $B=B_{1} U(\tau)$.

The purpose of the section is to establish the following statement.

Proposition 5. Let $e \in \mathfrak{g}$ be a nilpotent element. Let $\left(S_{0}, \tau\right)$ be as above and let $B \subset G$ be a Borel subgroup adapted to the pair $\left(S_{0}, \tau\right)$ in the sense of Definition 4 . Let $S=S_{0} \tau\left(\mathbb{K}^{*}\right)$.

Let $P \subset G$ be a parabolic subgroup, $V \subset \mathfrak{g}$ be a $P$-stable subspace, and consider the variety $\mathcal{P}_{e, V}=\left\{g P \in G / P: g^{-1} \cdot e \in V\right\}$ as in Definition 1 , Let $X$ denote either the whole variety $\mathcal{P}_{e, V}$ or a smooth irreducible component of $\mathcal{P}_{e, V}$. Let $B x_{0} \subset G / P$ be a $B$-orbit, with $x_{0} \in G / P$, and let $\left(B x_{0}\right)^{S}$ denote the subset of points fixed by $S$.

(a) If $X \cap\left(B x_{0}\right) \neq \emptyset$, then $X \cap\left(B x_{0}\right)^{S} \neq \emptyset$.

(b) Moreover, in this case, there is a map

$$
\rho: X \cap\left(B x_{0}\right) \rightarrow X \cap\left(B x_{0}\right)^{S}
$$

whose restriction $\rho^{-1}(C) \rightarrow C$ over each connected component $C \subset X \cap$ $\left(B x_{0}\right)^{S}$ is an algebraic affine bundle.

(c) In particular, if $X \cap\left(B x_{0}\right)^{S}$ is isomorphic to an affine space, then so is $X \cap\left(B x_{0}\right)$.

The proof is given in the following subsections. It is inspired by [7, §3] and [11, $\S 11]$.

4.1. A preliminary lemma. In this subsection, $\sigma: \mathbb{K}^{*} \rightarrow G$ denotes a cocharacter and $Q(\sigma), L(\sigma), U(\sigma)$ are the parabolic subgroup, Levi subgroup, and unipotent radical corresponding to $\sigma$ in the sense of (1)-(3). Let $B, T \subset G$ be a Borel subgroup and a maximal torus such that

$$
\sigma\left(\mathbb{K}^{*}\right) \subset T \subset B \subset Q(\sigma) .
$$

Let $P \subset G$ be another parabolic subgroup. It is well known that the flag variety $G / P$ consists of finitely many $B$-orbits (each one comprising a unique $T$-fixed point) and so of finitely many $Q(\sigma)$-orbits. For a $\sigma\left(\mathbb{K}^{*}\right)$-stable subset $X \subset G / P$, we denote by $X^{\sigma}$ the corresponding subset of $\sigma\left(\mathbb{K}^{*}\right)$-fixed points. The map

$$
r_{\sigma}: G / P \rightarrow(G / P)^{\sigma}, g P \mapsto \lim _{t \rightarrow 0} \sigma(t) g P
$$

is well defined.

Lemma 5. Let $Q(\sigma) x_{0} \subset G / P$ be a $Q(\sigma)$-orbit with $x_{0} \in(G / P)^{T}$.

(a) The fixed point set $\left(Q(\sigma) x_{0}\right)^{\sigma}$ consists of the single $L(\sigma)$-orbit $L(\sigma) x_{0}$ and is a projective variety (in particular it is a connected component of $(G / P)^{\sigma}$ and each connected component of $(G / P)^{\sigma}$ is of this form). 
(b) $r_{\sigma}$ restricts to an algebraic affine bundle $Q(\sigma) x_{0} \rightarrow\left(Q(\sigma) x_{0}\right)^{\sigma}$; moreover, for every $q \in Q(\sigma)$, writing $q=\ell u$ where $\ell \in L(\sigma)$ and $u \in U(\sigma)$, we have $r_{\sigma}\left(q x_{0}\right)=\ell x_{0}$

(c) $r_{\sigma}\left(B x_{0}\right)=\left(B x_{0}\right)^{\sigma}$.

Proof. Let $q=\ell u \in Q(\sigma)$ with $\ell \in L(\sigma)$ and $u \in U(\sigma)$. We first check the relation

$$
r_{\sigma}\left(q x_{0}\right)=\ell x_{0} \text {. }
$$

Since $\sigma(t) \ell=\ell \sigma(t)$ and $\sigma(t) x_{0}=x_{0}$, we obtain

$$
\sigma(t) q x_{0}=\ell \sigma(t) u \sigma(t)^{-1} x_{0} \text { for all } t \in \mathbb{K}^{*} .
$$

Relation (5) then follows by letting $t \rightarrow 0$ (and invoking (2) ).

The first claim in part (a) of the lemma is a consequence of relation (5) and the fact that the restriction of $r_{\sigma}$ to $\left(Q(\sigma) x_{0}\right)^{\sigma}$ is the identity. It implies that $\left(Q(\sigma) x_{0}\right)^{\sigma}$ is isomorphic to the quotient $L(\sigma) / M_{0}$ where $M_{0}=\left\{g \in L(\sigma): g x_{0}=x_{0}\right\}$. Write $x_{0}=g_{0} P$ with $g_{0} \in G$. An element $g \in G$ satisfies $g x_{0}=x_{0}$ if and only if $g \in g_{0} P g_{0}^{-1}$. Thus $M_{0}=L(\sigma) \cap\left(g_{0} P g_{0}^{-1}\right)$. The fact that $x_{0}$ is fixed by $\sigma\left(\mathbb{K}^{*}\right)$ yields $\sigma\left(\mathbb{K}^{*}\right) \subset g_{0} P g_{0}^{-1}$. Since $L(\sigma)=Z_{G}(\sigma)$, the last inclusion implies that $M_{0}$ is a parabolic subgroup of $L(\sigma)$, hence $L(\sigma) / M_{0}$ is a projective variety. This completes the proof of part (a).

Relation (5) establishes the second claim in part (b) of the lemma. It follows from Białynicki-Birula's theorem (see Theorem 3 below) that the map $r_{\sigma}$ restricts to an algebraic affine bundle $r_{\sigma}^{-1}(C) \rightarrow C$ for every connected component $C \subset(G / P)^{\sigma}$. Now (5) implies that $r_{\sigma}^{-1}\left(\left(Q(\sigma) x_{0}\right)^{\sigma}\right)=Q(\sigma) x_{0}$. Whence the first claim in part (b) of the lemma.

The inclusion $B \subset Q(\sigma)$ yields the inclusion $U(\sigma) \subset B$. Thereby, every element in $B$ can be written $b=\ell u$ with $\ell \in L(\sigma) \cap B$ and $u \in U(\sigma) \subset B$. By (5), we have $r_{\sigma}\left(b x_{0}\right)=\ell x_{0} \in\left(B x_{0}\right)^{\sigma}$, whence $r_{\sigma}\left(B x_{0}\right)=\left(B x_{0}\right)^{\sigma}$. This establishes part (c) of the statement.

The proof uses the following result, which is a weak version of [1, Theorem 4.1].

Theorem 3. Let $X$ be a smooth, projective variety equipped with an algebraic action of $\mathbb{K}^{*}$, so giving rise to the retraction $r_{X}: X \rightarrow X^{\mathbb{K}^{*}}, x \mapsto \lim _{t \rightarrow 0} t \cdot x$, where $X^{\mathbb{K}^{*}} \subset X$ denotes the subset of $\mathbb{K}^{*}$-fixed points. Let $Y \subset X$ be a smooth, $\mathbb{K}^{*}$-stable, locally closed subvariety and assume that $r_{X}(Y)=Y^{\mathbb{K}^{*}}:=Y \cap X^{\mathbb{K}^{*}}$. Then, $Y^{\mathbb{K}^{*}}$ is smooth and, for every connected component $C \subset Y^{\mathbb{K}^{*}}$, the restriction $Y \cap r_{X}^{-1}(C) \rightarrow C$ of $r_{X}$ is an algebraic affine bundle.

Proof. The smoothness of $Y^{\mathbb{K}^{*}}$ is a consequence of the facts that $Y$ is smooth and that the group $\mathbb{K}^{*}$ is linearly reductive (see [11, Theorem 11.7]). Then, the assumptions allow us to apply [1, Theorem 4.1], which yields an algebraic affine bundle $\gamma: Y \cap r_{X}^{-1}(C) \rightarrow C$, fulfilling certain additional properties. The fact that $\gamma$ coincides with the restriction of $r_{X}$ is explained in [11, $\left.\S \S 11.15-16\right]$.

4.2. First step of the proof of Proposition 5. In this subsection, we show the following statement.

Proposition 6. Same notation as in Proposition 5 .

(a) Assume that $\mathcal{P}_{e, V} \cap\left(Q(\tau) x_{0}\right) \neq \emptyset$. Then, $\mathcal{P}_{e, V} \cap\left(Q(\tau) x_{0}\right)^{\tau}$ is nonempty, smooth, and the retraction $r_{\tau}$ (see (40) restricts to a well-defined map

$$
r_{\tau}^{\prime}: \mathcal{P}_{e, V} \cap\left(Q(\tau) x_{0}\right) \rightarrow \mathcal{P}_{e, V} \cap\left(Q(\tau) x_{0}\right)^{\tau}
$$


whose restriction $r_{\tau}^{\prime-1}(C) \rightarrow C$ over each connected component $C \subset \mathcal{P}_{e, V} \cap$ $\left(Q(\tau) x_{0}\right)^{\tau}$ is an algebraic affine bundle.

(b) Assume that $X \cap\left(B x_{0}\right) \neq \emptyset$. Then, $X \cap\left(B x_{0}\right)^{\tau} \neq \emptyset$, and $r_{\tau}$ restricts to a well-defined map $X \cap\left(B x_{0}\right) \rightarrow X \cap\left(B x_{0}\right)^{\tau}$ whose restriction over each connected component of $X \cap\left(B x_{0}\right)^{\tau}$ is an algebraic affine bundle.

The proof relies on Lemma 5 and on the following fact, which is a reformulation of [7, Proposition 3.2].

Lemma 6. Same notation as in Proposition 6. The variety $\mathcal{P}_{e, V} \cap\left(Q(\tau) x_{0}\right)$ is smooth.

Proof of Lemma 6. Write $x_{0}=g_{0} P$. Consider the closed subset of $Q(\tau)$ given by

$$
A=\left\{q \in Q(\tau): q^{-1} \cdot e \in g_{0} \cdot V\right\} .
$$

By definition of $A$, the map

$$
A \rightarrow \mathcal{P}_{e, V} \cap\left(Q(\tau) x_{0}\right), q \mapsto q x_{0}
$$

is well defined, surjective, and smooth. We also consider the map

$$
A \rightarrow\left(g_{0} \cdot V\right) \cap(Q(\tau) \cdot e), q \mapsto q^{-1} \cdot e .
$$

Again, the definition of $A$ ensures that this map is well defined, surjective and, moreover, it is smooth. Note that $\left(g_{0} \cdot V\right) \cap(Q(\tau) \cdot e)$ is an open subvariety of $\left(g_{0} \cdot V\right) \cap \overline{Q(\tau) \cdot e}$, which is a smooth variety, as the intersection of two linear subspaces of $\mathfrak{g}$ (by Proposition $4(\mathrm{a}))$. Thereby, the varieties $\left(g_{0} \cdot V\right) \cap(Q(\tau) \cdot e), A$, and $\mathcal{P}_{e, V} \cap\left(Q(\tau) x_{0}\right)$ are smooth.

Proof of Proposition 6. We first emphasize properties of the map $r_{\tau}$ :

$$
r_{\tau}\left(Q(\tau) x_{0}\right)=\left(Q(\tau) x_{0}\right)^{\tau}, \quad r_{\tau}\left(B x_{0}\right)=\left(B x_{0}\right)^{\tau},
$$

and

$$
r_{\tau}\left(\mathcal{P}_{e, V}\right) \subset \mathcal{P}_{e, V}
$$

Relation (6) follows from Lemma 5, whereas relation (77) follows from the fact that $\mathcal{P}_{e, V}$ is closed in $G / P$ and stable by the action $(t, x) \mapsto \tau(t) x$ (the last fact is a consequence of Definition 3(a) and the definition of the variety $\mathcal{P}_{e, V}$ in Definition 11). Combining the first part of (6) with (7), we obtain

$$
r_{\tau}\left(\mathcal{P}_{e, V} \cap\left(Q(\tau) x_{0}\right)\right)=\mathcal{P}_{e, V} \cap\left(Q(\tau) x_{0}\right)^{\tau} .
$$

Lemma 6 and relation (8) allow us to apply Theorem [3, which yields part (a) of the proposition.

If $X$ is a smooth irreducible component of $\mathcal{P}_{e, V}$, then it is also stable by the action $(t, x) \mapsto \tau(t) x$, i.e.,

$$
r_{\tau}(X) \subset X,
$$

and it follows from Theorem 3 that the restriction

$$
X \rightarrow X^{\tau}, x \mapsto \lim _{t \rightarrow 0} \tau(t) x
$$

(still denoted $r_{\tau}^{\prime}$ ) of $r_{\tau}$ is an algebraic affine bundle over each connected component of $X^{\tau}$. In what follows, let $X$ denote (as in the statement) either the whole variety 
$\mathcal{P}_{e, V}$ or a smooth irreducible component of $\mathcal{P}_{e, V}$. Combining the second relation in (6) with (7) or (9), we get

$$
r_{\tau}^{\prime}\left(X \cap\left(B x_{0}\right)\right)=X \cap\left(B x_{0}\right)^{\tau} .
$$

Whence $X \cap\left(B x_{0}\right)^{\tau} \neq \emptyset$ whenever $X \cap\left(B x_{0}\right) \neq \emptyset$. Actually, since the orbit $B x_{0}$ is arbitrary, it also follows that

$$
r_{\tau}^{\prime-1}\left(X \cap\left(B x_{0}\right)^{\tau}\right)=X \cap\left(B x_{0}\right) .
$$

Part (b) of the proposition now follows from part (a) and relation (10).

4.3. Second step of the proof of Proposition 5. In addition to the notation introduced at the beginning of Section 4 , we consider:

- $Q_{0} \subset G$ a parabolic subgroup admitting $G_{0}$ as Levi factor, such that $Q_{0} \cap$ $L(\tau)$ contains $B \cap L(\tau)$ (see Definition (4);

- $\tau_{0}: \mathbb{K}^{*} \rightarrow G$ a cocharacter corresponding to $G_{0}, Q_{0}$, and its unipotent radical $U_{0}$ in the sense of relations (11)-(3); that is,

$$
\begin{aligned}
& Q_{0}=\left\{g \in G: \lim _{t \rightarrow 0} \tau_{0}(t) g \tau_{0}(t)^{-1} \text { exists }\right\}, \\
& U_{0}=\left\{g \in G: \lim _{t \rightarrow 0} \tau_{0}(t) g \tau_{0}(t)^{-1}=1_{G}\right\}, \\
& G_{0}=Z_{G}\left(\tau_{0}\right) .
\end{aligned}
$$

Lemma 7. Every $b \in B$ can be written $b=b_{1} u_{1} u_{2}$, where $b_{1} \in B \cap L(\tau) \cap G_{0}$, $u_{1} \in L(\tau) \cap U_{0}$, and $u_{2} \in U(\tau)$ (where $U(\tau)$ denotes the unipotent radical of $Q(\tau)$ ).

Proof. Since $b \in B \subset Q(\tau)$, there are $b_{2} \in L(\tau)$ and $u_{2} \in U(\tau)$ such that $b=b_{2} u_{2}$. So, $b_{2}=b u_{2}^{-1} \in B U(\tau) \subset B$, hence $b_{2} \in B \cap L(\tau) \subset Q_{0} \cap L(\tau)$. Since $Q_{0}$ contains the torus $\tau\left(\mathbb{K}^{*}\right)$, the intersection $Q_{0} \cap L(\tau)$ is a parabolic subgroup of $L(\tau)$, with Levi decomposition

$$
Q_{0} \cap L(\tau)=\left(G_{0} \cap L(\tau)\right)\left(U_{0} \cap L(\tau)\right) .
$$

Thereby, there exist $b_{1} \in G_{0} \cap L(\tau)$ and $u_{1} \in U_{0} \cap L(\tau)$ such that $b_{2}=b_{1} u_{1}$. Whence $b_{1}=b_{2} u_{1}^{-1} \in B\left(U_{0} \cap L(\tau)\right) \subset B$. The proof is complete.

\section{Lemma 8.}

(a) Given $S \subset G$ a torus, there exists a cocharacter $\sigma: \mathbb{K}^{*} \rightarrow S$ such that $Z_{G}(S)=Z_{G}(\sigma)$.

(b) Let $S_{1}, S_{2} \subset G$ be tori such that $Z_{G}\left(S_{1}\right)=Z_{G}\left(S_{2}\right)=$ : L, then for every parabolic subgroup $P \subset G$, we have $(G / P)^{S_{1}}=(G / P)^{S_{2}}$.

Proof. (a) The cocharacter $\sigma$ can be constructed by arguing as in [11, §11.11] and [19, §8.4.5].

(b) The assumption shows in particular that $S:=S_{1} S_{2}$ is a torus of $L$. Applying part (a) we find a cocharacter $\sigma: \mathbb{K}^{*} \rightarrow S_{1}$ such that $L=Z_{G}(\sigma)$. Thus, $(G / P)^{\sigma} \supset$ $(G / P)^{S_{1}} \supset(G / P)^{S}$. Let $Q(\sigma) \subset G$ be the parabolic subgroup associated to $\sigma$ in the sense of (1) and let $Q(\sigma) x_{0} \subset G / P$ be a $Q(\sigma)$-orbit. By the Bruhat decomposition, we know that the fixed point set $\left(Q(\sigma) x_{0}\right)^{S}$ is nonempty, and we have the inclusion $\left(Q(\sigma) x_{0}\right)^{S} \subset\left(Q(\sigma) x_{0}\right)^{\sigma}$. Actually $\left(Q(\sigma) x_{0}\right)^{S}$ is $L$-stable whereas $\left(Q(\sigma) x_{0}\right)^{\sigma}$ is a single $L$-orbit (see Lemma $5(\mathrm{a})$ ), whence the equality $\left(Q(\sigma) x_{0}\right)^{S}=\left(Q(\sigma) x_{0}\right)^{\sigma}$. Since this holds for all $Q(\sigma)$-orbit, we obtain $(G / P)^{\sigma}=(G / P)^{S_{1}}=(G / P)^{S}$. Similarly, we have $(G / P)^{S_{2}}=(G / P)^{S}$, therefore $(G / P)^{S_{1}}=(G / P)^{S_{2}}$. 
Applying Lemma 8 in our case, since $G_{0}=Z_{G}\left(\tau_{0}\right)=Z_{G}\left(S_{0}\right)$, we obtain that

$$
(G / P)^{\tau_{0}}=(G / P)^{S_{0}} .
$$

We consider the retraction

$$
r_{\tau_{0}}: G / P \rightarrow(G / P)^{S_{0}}, x \mapsto \lim _{t \rightarrow 0} \tau_{0}(t) x .
$$

In this subsection, we establish the following proposition.

Proposition 7. Same notation as in Proposition 5. The map $r_{\tau_{0}}$ restricts to a well-defined map

$$
X \cap\left(B x_{0}\right)^{\tau} \rightarrow X \cap\left(B x_{0}\right)^{S_{0} \tau\left(\mathbb{K}^{*}\right)},
$$

whose restriction over each connected component of $X \cap\left(B x_{0}\right)^{S_{0} \tau\left(\mathbb{K}^{*}\right)}$ is an algebraic affine bundle.

Proof. Since the cocharacter $\tau_{0}$ has values in the center of $G_{0}$ and $e$ is an element of $\operatorname{Lie}\left(G_{0}\right)$, we see that $\tau_{0}$ stabilizes $e$, which implies that the variety $\mathcal{P}_{e, V}$ is stable by the action $(t, x) \mapsto \tau_{0}(t) x$, as well as $X$ whenever $X$ is an irreducible component of $\mathcal{P}_{e, V}$. The fact that $G_{0}=Z_{G}\left(\tau_{0}\right)$ also implies that the tori $\tau_{0}\left(\mathbb{K}^{*}\right), \tau\left(\mathbb{K}^{*}\right)$ commute, hence $\tau_{0}\left(\mathbb{K}^{*}\right) \subset L(\tau)$, which implies that $\left(Q(\tau) x_{0}\right)^{\tau}$ is also stabilized by the action $(t, x) \mapsto \tau_{0}(t) x$. We conclude that $\tau_{0}$ induces an algebraic action of $\mathbb{K}^{*}$ on the variety $\mathcal{P}_{e, V} \cap\left(Q(\tau) x_{0}\right)^{\tau}$, as well as on the fixed point set $X^{\tau}$ whenever $X \subset \mathcal{P}_{e, V}$ is a component.

It follows from Lemma 5 (a) and Proposition 6 that the variety $\mathcal{P}_{e, V} \cap\left(Q(\tau) x_{0}\right)^{\tau}$ is smooth and projective. By Theorem 3 , the map $r_{\tau_{0}}$ restricts to a map

$$
\mathcal{P}_{e, V} \cap\left(Q(\tau) x_{0}\right)^{\tau} \rightarrow \mathcal{P}_{e, V} \cap\left(Q(\tau) x_{0}\right)^{S_{0} \tau\left(\mathbb{K}^{*}\right)},
$$

which is an algebraic affine bundle over each connected component of the right-hand side. If $X$ is a smooth component of $\mathcal{P}_{e, V}$, then the fixed point set $X^{\tau}$ is smooth and projective. By Theorem $3, r_{\tau_{0}}$ restricts to a map

$$
X^{\tau} \rightarrow X^{S_{0} \tau\left(\mathbb{K}^{*}\right)},
$$

which is an algebraic affine bundle over each connected component of $X^{S_{0} \tau\left(\mathbb{K}^{*}\right)}$. In order to complete the proof of the proposition, it then suffices to check the following equality:

$$
r_{\tau_{0}}^{-1}\left(\left(B x_{0}\right)^{S_{0} \tau\left(\mathbb{K}^{*}\right)}\right) \cap(G / P)^{\tau}=\left(B x_{0}\right)^{\tau}
$$

The orbit $B x_{0}$ being arbitrary, (14) ensues once we show

$$
r_{\tau_{0}}\left(\left(B x_{0}\right)^{\tau}\right) \subset\left(B x_{0}\right)^{S_{0} \tau\left(\mathbb{K}^{*}\right)} .
$$

Let us check (15). First, we know from the Bruhat decomposition that the fixed point set $\left(B x_{0}\right)^{S_{0} \tau\left(\mathbb{K}^{*}\right)}$ is nonempty, hence we may assume without any loss of generality that $x_{0}$ is a $S_{0} \tau\left(\mathbb{K}^{*}\right)$-fixed point. Let $x \in\left(B x_{0}\right)^{\tau}$, thus there is $b \in B$ such that $x=b x_{0}$. Write $b=b_{1} u_{1} u_{2}$, where $b_{1} \in B \cap G_{0} \cap L(\tau), u_{1} \in U_{0} \cap L(\tau)$, and $u_{2} \in U(\tau)$ (see Lemma 7 ). The facts that $x$ is a $\tau\left(\mathbb{K}^{*}\right)$-fixed point and that $x_{0}$ is a $S_{0} \tau\left(\mathbb{K}^{*}\right)$-fixed point yield

$$
x=\tau(t) x=b_{1} u_{1} \tau(t) u_{2} \tau(t)^{-1} x_{0} \text { for all } t \in \mathbb{K}^{*},
$$

hence, letting $t \rightarrow 0$ (and using (2) ), we get $x=b_{1} u_{1} x_{0}$. Similarly,

$$
\tau_{0}(t) x=b_{1} \tau_{0}(t) u_{1} \tau_{0}(t)^{-1} x_{0} \text { for all } t \in \mathbb{K}^{*},
$$


so letting $t \rightarrow 0$ we obtain

$$
r_{\tau_{0}}(x)=\lim _{t \rightarrow 0} \tau_{0}(t) x=b_{1} x_{0} \in\left(B x_{0}\right)^{S_{0} \tau\left(\mathbb{K}^{*}\right)}
$$

(see (12) ). Whence (15). The proof of the proposition is now complete.

Proposition 5 is finally obtained by combining Proposition [6(b) and Proposition 7

Remark 2. As noted by the referee, in the case where $X=\mathcal{P}_{e, V}$, Proposition 5 can be demonstrated more directly by first showing that $X \cap\left(B x_{0}\right)$ is smooth (as in [15. Proposition 3.7 and Corollary 4.9]) and then applying [7, §1.5] (or Theorem [3).

\section{Proof of the MAin Results}

This section contains the proofs of the main results stated in Section 1. Some preliminaries are needed.

5.1. Preliminary lemmas. We start with two general lemmas.

Lemma 9. Let $S \subset G$ be a torus and let $L=Z_{G}(S)$ be the corresponding Levi subgroup. Let $H \subset L$ be a subgroup. The following conditions are equivalent:

(i) the number of $H S$-fixed points in the flag variety $G / P$ is finite, for all parabolic subgroups $P \subset G$;

(ii) the number of $H$-fixed points in the flag variety $L / P_{0}$ is finite, for all parabolic subgroups $P_{0} \subset L$.

Proof. Let $Q \subset G$ be a parabolic subgroup admitting $L$ as a Levi factor. Let $P \subset G$ be an arbitrary parabolic subgroup. The flag variety $G / P$ admits finitely many $Q$-orbits and each one is of the form $Q g P$ where $g P$ is an $S$-fixed point, i.e., $S \subset g P g^{-1}$. The last fact implies that $P_{0}:=L \cap\left(g P g^{-1}\right)$ is a parabolic subgroup of $L$ (and each parabolic subgroup of $L$ can be obtained in this way, for appropriate $P$, $g$ ). From Lemma 5(a) and Lemma 8, we know that there is a natural isomorphism

$$
(Q g P)^{S}=L g P \rightarrow L / P_{0} .
$$

This map being $L$-equivariant, it yields an isomorphism between fixed point sets

$$
(Q g P)^{H S} \cong\left(L / P_{0}\right)^{H} \text {. }
$$

The parabolic subgroups $P \subset G$ and $P_{0} \subset L$ being arbitrary, we get the equivalence between (ii) and:

(i') For every parabolic subgroup $P \subset G$, there is a finite number of $H S$-fixed points in each $Q$-orbit of the flag variety $G / P$.

Since there are finitely many $Q$-orbits in any flag variety $G / P$, we know that (i) and (i') are equivalent. This completes the proof.

Lemma 10. Let $\sigma: \mathbb{K}^{*} \rightarrow G$ be a cocharacter. The following conditions are equivalent:

(i) The cocharacter $\sigma$ is regular (i.e., $Z_{G}(\sigma)$ is a maximal torus).

(ii) For every parabolic subgroup $P \subset G$, the fixed point set $(G / P)^{\sigma}$ is finite.

Proof. Applying Lemma 9 to the groups $S=\sigma\left(\mathbb{K}^{*}\right)$ and $H=\left\{1_{G}\right\}$, we see that (ii) is equivalent to:

(i') the flag variety $Z_{G}(\sigma) / P_{0}$ is a finite set for all parabolic subgroups $P_{0} \subset$ $Z_{G}(\sigma)$. 
Condition (i') is clearly equivalent to saying that the Levi subgroup $Z_{G}(\sigma)$ is a (necessarily maximal) torus, hence it is equivalent to (i).

The next lemma is a key argument in the proof of Proposition 1 and Theorem 1 , This is also the first place where the assumption that $e$ is of standard Levi type is invoked (the results of the previous section hold without this assumption).

Lemma 11. Let $e \in \mathfrak{g}$ be a nilpotent element. Let $S_{0} \subset Z_{G}(e)$ be a maximal torus and let $G_{0}=Z_{G}\left(S_{0}\right)$ be the corresponding Levi subgroup, so satisfying that $e$ is a distinguished nilpotent element of $\operatorname{Lie}\left(G_{0}\right)$. Let $\tau: \mathbb{K}^{*} \rightarrow G$ be a cocharacter associated to $e$ such that $\tau\left(\mathbb{K}^{*}\right) \subset G_{0}^{\prime}$. Assume that $e$ is a nilpotent element of standard Levi type. Then the torus $S_{0} \tau\left(\mathbb{K}^{*}\right)$ has a finite number of fixed points in the flag variety $G / P$, for all parabolic subgroups $P \subset G$.

Proof. By assumption, $e$ is a regular nilpotent element of $\operatorname{Lie}\left(G_{0}\right)$. Note that $\tau$ is also a cocharacter associated to $e$ in the group $G_{0}$. Then, from Lemma 3, we have that $\tau$ is a regular cocharacter of $G_{0}$. Hence, for every parabolic subgroup $P_{0} \subset G_{0}$, the fixed point set $\left(G_{0} / P_{0}\right)^{\tau}$ is finite (see Lemma 10). Applying Lemma 9 to the torus $S=S_{0}$ and the group $H=\tau\left(\mathbb{K}^{*}\right)$, we deduce that the fixed point set $(G / P)^{S_{0} \tau\left(\mathbb{K}^{*}\right)}$ is finite for all parabolic subgroups $P \subset G$.

5.2. Proof of Proposition 1. The implication (i) $\Rightarrow$ (ii) is a consequence of Lemmas 8 , 10, and 11]

Before proving the inverse implication, we do a preliminary construction. Let $S_{0}$ be a maximal torus of $Z_{G}(e)$ and let $\tau: \mathbb{K}^{*} \rightarrow Z_{G}\left(S_{0}\right)$ be a cocharacter associated to the nilpotent element $e$, then it follows from Lemma 1 that

$$
S_{0} \tau\left(\mathbb{K}^{*}\right) \text { is a maximal torus of } N_{G}(e) .
$$

Now let us show the implication (ii) $\Rightarrow(\mathrm{i})$. Assume that the group $N_{G}(e)$ contains a regular rank one subtorus, the image of a regular cocharacter $\eta: \mathbb{K}^{*} \rightarrow G$. The torus $\eta\left(\mathbb{K}^{*}\right)$ lies in a maximal torus of $N_{G}(e)$. Since all the maximal tori of $N_{G}(e)$ are conjugate, up to replacing $\eta$ by a suitable conjugate, we may assume that $\eta\left(\mathbb{K}^{*}\right) \subset S_{0} \tau\left(\mathbb{K}^{*}\right)$. It follows from Lemma 10 that the torus $S_{0} \tau\left(\mathbb{K}^{*}\right)$ has a finite number of fixed points in the flag variety $G / P$, for all parabolic subgroups $P \subset G$. Invoking Lemma 9 with $S=S_{0}$ and $H=\tau\left(\mathbb{K}^{*}\right)$, and again applying Lemma 10, we obtain that $\tau$ is a regular cocharacter of $Z_{G}\left(S_{0}\right)$. Finally, from Lemma 3, we conclude that $e$ is a regular element of $\operatorname{Lie}\left(Z_{G}\left(S_{0}\right)\right)$, hence a nilpotent element of standard Levi type in $\mathfrak{g}$. The proof of Proposition 1 is complete.

5.3. Proof of Theorem 1. We establish the following more precise statement.

Theorem 4. Let $e \in \mathfrak{g}$ be a nilpotent element of standard Levi type. Let $S_{0}$ be a maximal torus of $Z_{G}(e)$ and let $\tau: \mathbb{K}^{*} \rightarrow G_{0}:=Z_{G}\left(S_{0}\right)$ be a cocharacter associated to e. Finally, let $B \subset G$ be a Borel subgroup adapted to $\left(S_{0}, \tau\right)$ in the sense of Definition 4. Then, for every parabolic subgroup $P \subset G$ and every $P$-stable subspace $V \subset \mathfrak{g}$, denoting by $X$ either the whole variety $\mathcal{P}_{e, V}$ or a smooth irreducible component of $\mathcal{P}_{e, V}$, the following holds true: for every $B$-orbit $B x_{0} \subset G / P$, the intersection $X \cap\left(B x_{0}\right)$ is either empty or isomorphic to an affine space.

Proof of Theorem 4. In view of Proposition 5(c), the proof reduces to show that $X \cap\left(B x_{0}\right)^{S}$ is isomorphic to an affine space, where $S=S_{0} \tau\left(\mathbb{K}^{*}\right)$. We claim that 
$X \cap\left(B x_{0}\right)^{S}$, if nonempty, is a singleton, actually we claim that

$$
\left(B x_{0}\right)^{S} \text { is a singleton. }
$$

Clearly, the proof is complete once we show (17). By Lemma 11, using also Lemmas 8 and 10, the group $T:=Z_{G}(S)$ is a maximal torus of $G$ (contained in $B$ ). In particular $Z_{G}(T)=Z_{G}(S)$. Invoking Lemma 8(b), we deduce that

$$
(G / P)^{S}=(G / P)^{T} \text {. }
$$

By the Bruhat decomposition, every $B$-orbit of $G / P$ comprises a unique $T$-fixed point. Whence (17). The proof of the theorem is complete.

\section{EXAMPLES}

We illustrate the previous results in the cases of the general linear group $G=$ $G L(V)$ and of the symplectic group $G=S p(V, \omega)$. In each case, we explain the construction of an explicit Borel subgroup adapted to a nilpotent element of standard Levi type.

6.1. Case of the general linear group. Let $V$ be a vector space of finite dimension $n \geq 1$. Consider the group $G=G L(V)$. Its Lie algebra $\mathfrak{g}=\operatorname{End}(V)$ coincides with the space of linear endomorphisms of $V$. A nilpotent element is then an endomorphism $e \in \operatorname{End}(V)$, which is nilpotent in the usual sense.

The lengths $\pi=\left(\pi_{1} \geq \pi_{2} \geq \ldots \geq \pi_{k}\right)$ of the Jordan blocks of $e$ form a partition of $n$. Represent the partition $\pi$ by a Young diagram and view this Young diagram as a set of boxes, still denoted by $\pi$, where $\pi_{i}$ denotes the subset of boxes in the $i$-th row. Consider a basis $\left\{v_{a}: a \in \pi\right\}$ parametrized by $\pi$, such that:

- $e\left(v_{a}\right)=0$ if $a$ belongs to the first column of $\pi$;

- $e\left(v_{a}\right)=v_{a^{\prime}}$ where $a^{\prime}$ denotes the box next to $a$ on the left, otherwise.

Thus $\left\{v_{a}\right\}$ is a Jordan basis for the endomorphism $e$, with the $i$-th Jordan block $V_{i}:=\left\langle v_{a}: a \in \pi_{i}\right\rangle_{\mathbb{K}}$ corresponding to the boxes in the $i$-th row of $\pi$.

Given $\left(t_{1}, \ldots, t_{k}\right) \in\left(\mathbb{K}^{*}\right)^{k}$, define $h\left(t_{1}, \ldots, t_{k}\right) \in G L(V)$ by $v_{a} \mapsto t_{i} v_{a}$ whenever $a \in \pi_{i}$ and set $S_{0}=\left\{h\left(t_{1}, \ldots, t_{k}\right)\right\} \cong\left(\mathbb{K}^{*}\right)^{k}$. Then, $S_{0}$ is a maximal torus of $Z_{G}(e)$. The corresponding Levi subgroup is $G_{0}:=Z_{G}\left(S_{0}\right)=\prod_{i=1}^{k} G L\left(V_{i}\right)$ and its derived group is $G_{0}^{\prime}=\prod_{i=1}^{k} S L\left(V_{i}\right)$.

Next, we construct a cocharacter associated to $e$. To do this, we attribute a weight $\nu(a) \in \mathbb{Z}$ to each box $a \in \pi$ as follows. Suppose $a \in \pi_{i}$, and let

$$
\nu(a)=\#\left\{\text { boxes of } \pi_{i} \text { on the right of } a\right\}-\#\left\{\text { boxes of } \pi_{i} \text { on the left of } a\right\},
$$

where \#A denotes the cardinal of a set $A$. Then, we define $\tau: \mathbb{K}^{*} \rightarrow G L(V)$ by

$$
\tau(t): v_{a} \mapsto t^{\nu(a)} v_{a} \text { for all } a \in \pi .
$$

It is readily seen that we have $\tau(t) e \tau(t)^{-1}=t^{2} e$ and the image of $\tau$ is contained in $G_{0}^{\prime}$, hence $\tau$ is a cocharacter associated to $e$.

Finally, a Borel subgroup adapted to $\left(S_{0}, \tau\right)$ can be obtained as follows. Fix a permutation $r=\left(r_{1}, \ldots, r_{k}\right)$ of $(1, \ldots, k)$ and consider the ordering $\prec$ of the rows of $\pi$ defined by $r$, that is, let $\pi_{r_{1}} \prec \ldots \prec \pi_{r_{k}}$. There is a unique total order $<$ on the boxes of the diagram $\pi$ such that:

- the weight $\nu$ is nonincreasing: if $a<a^{\prime}$, then $\nu(a) \geq \nu\left(a^{\prime}\right)$;

- if $a \in \pi_{i}$ and $a^{\prime} \in \pi_{j}$ have the same weight, then $a<a^{\prime} \Leftrightarrow \pi_{i} \prec \pi_{j}$. 
Denote by $a_{1}, \ldots, a_{n}$ the boxes of $\pi$ enumerated according to this order, i.e., so that $a_{1}<\ldots<a_{n}$. Define $B \subset G L(V)$ as the subgroup of the automorphisms that are upper triangular in the basis $\left(v_{a_{1}}, \ldots, v_{a_{n}}\right)$. Then, $B$ is a Borel subgroup adapted to $\left(S_{0}, \tau\right)$ in the sense of Definition 4 . Note that different Borel subgroups can be obtained, depending on the choice of the permutation $r$.

Example 3. Let $e \in \operatorname{End}\left(\mathbb{K}^{7}\right)$ be a nilpotent endomorphism with three Jordan blocks of lengths $(4,2,1)$. Its Jordan form can be represented by the Young diagram:

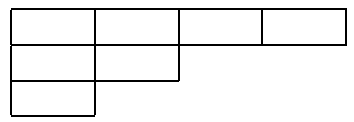

The following tableau indicates the weight $\nu(a)$ for each box of the diagram:

\begin{tabular}{|c|c|c|c|}
\hline 3 & 1 & -1 & -3 \\
\hline 1 & -1 & \multicolumn{2}{|c}{} \\
\cline { 1 - 2 } 0 & \multicolumn{2}{|c}{} \\
\cline { 1 - 2 } &
\end{tabular}

Consider the natural ordering of the rows from top to bottom (i.e., $\left(r_{1}, r_{2}, r_{3}\right)=$ $(1,2,3))$. Then, the numbering of the boxes $a_{1}, \ldots, a_{7}$ constructed according to the procedure described above is as follows (we put $i$ in the box $a_{i}$ ):

\begin{tabular}{|l|l|l|l|}
\hline 1 & 2 & 5 & 7 \\
\hline 3 & 6 & \multicolumn{2}{|c}{} \\
\cline { 1 - 2 } 4 & \multicolumn{2}{|c}{} \\
\cline { 1 - 2 }
\end{tabular}

In the so-obtained basis $\left(v_{a_{1}}, \ldots, v_{a_{7}}\right)$, the matrix of $e$ becomes

$$
\left(\begin{array}{lllllll}
0 & 1 & 0 & 0 & 0 & 0 & 0 \\
0 & 0 & 0 & 0 & 1 & 0 & 0 \\
0 & 0 & 0 & 0 & 0 & 1 & 0 \\
0 & 0 & 0 & 0 & 0 & 0 & 0 \\
0 & 0 & 0 & 0 & 0 & 0 & 1 \\
0 & 0 & 0 & 0 & 0 & 0 & 0 \\
0 & 0 & 0 & 0 & 0 & 0 & 0
\end{array}\right)
$$

The tori $S_{0}$ and $\tau\left(\mathbb{K}^{*}\right)$ correspond to the subgroups of matrices of the form

$$
\left(\begin{array}{ccccccc}
t_{1} & 0 & 0 & 0 & 0 & 0 & 0 \\
0 & t_{1} & 0 & 0 & 0 & 0 & 0 \\
0 & 0 & t_{2} & 0 & 0 & 0 & 0 \\
0 & 0 & 0 & t_{3} & 0 & 0 & 0 \\
0 & 0 & 0 & 0 & t_{1} & 0 & 0 \\
0 & 0 & 0 & 0 & 0 & t_{2} & 0 \\
0 & 0 & 0 & 0 & 0 & 0 & t_{1}
\end{array}\right), \quad\left(\begin{array}{ccccccc}
t^{3} & 0 & 0 & 0 & 0 & 0 & 0 \\
0 & t & 0 & 0 & 0 & 0 & 0 \\
0 & 0 & t & 0 & 0 & 0 & 0 \\
0 & 0 & 0 & 1 & 0 & 0 & 0 \\
0 & 0 & 0 & 0 & t^{-1} & 0 & 0 \\
0 & 0 & 0 & 0 & 0 & t^{-1} & 0 \\
0 & 0 & 0 & 0 & 0 & 0 & t^{-3}
\end{array}\right) .
$$

Finally, the adapted Borel subgroup $B$ corresponds to the subgroup of upper triangular matrices.

Remark 3. The result in Theorem 1(b) concerns only smooth components of varieties of the form $\mathcal{P}_{e, V}$. However, [9, §6.2] points out a singular component $X$ of a Springer fiber $\mathcal{B}_{e}$ (corresponding to $e \in \operatorname{End}\left(\mathbb{K}^{7}\right)$ of Jordan form $(3,2,2)$ ), whose intersection with each $B$-orbit (for an adapted $B$ ) is isomorphic to an affine space.

6.2. Case of the symplectic group. Assume that the vector space $V$ has even dimension $n=2 p$ and is endowed with a symplectic form $\omega: V \times V \rightarrow \mathbb{K}$. Consider the symplectic group $G=S p(V, \omega)$ formed by the automorphisms $g \in G L(V)$ that preserve the symplectic form, i.e., $\omega\left(g v, g v^{\prime}\right)=\omega\left(v, v^{\prime}\right)$ for all $v, v^{\prime} \in V$. Its Lie algebra is the symplectic Lie algebra $\mathfrak{g}=\mathfrak{s p}(V, \omega)$ formed by the endomorphisms $x \in \operatorname{End}(V)$ that are antiadjoint with respect to $\omega$, i.e., $\omega\left(x v, v^{\prime}\right)+\omega\left(v, x v^{\prime}\right)=0$ 
for all $v, v^{\prime}$. The characteristic of the base field $\mathbb{K}$ is assumed to be different from 2 , so that it is good for $G$.

Remark 4. Recall that a possible interpretation of the elements of $S p(V, \omega)$ and $\mathfrak{s p}(V, \omega)$ in terms of matrices is as follows. Consider a basis $\left(v_{1}, \ldots, v_{n}\right)$ of $V$ such that

$$
\omega\left(v_{i}, v_{j}\right)=\left\{\begin{array}{ll}
1 & \text { if } i+j=n+1, \\
0 & \text { otherwise, }
\end{array} \text { for all } 1 \leq i \leq j \leq n .\right.
$$

Let $J_{p}$ denote the $p \times p$ sized matrix with 1's on the antidiagonal and 0's elsewhere, and let $K=\left(\begin{array}{cc}0 & J_{p} \\ -J_{p} & 0\end{array}\right) \in S L_{n}(\mathbb{K})$. Then, through the basis $\left(v_{1}, \ldots, v_{n}\right)$, the group $S p(V, \omega)$ interprets as the group of matrices $g \in S L_{n}(\mathbb{K})$ such that $\left(g^{\mathrm{t}}\right) K g=$ $K$, and the Lie algebra $\mathfrak{s p}(V, \omega)$ interprets as the space of matrices $x \in M_{n}(\mathbb{K})$ such that $\left(x^{\mathrm{t}}\right) K+K x=0$, that is, of the form $x=\left(\begin{array}{cc}P & R \\ S & -P^{\delta}\end{array}\right)$ with $P, R, S \in M_{p}(\mathbb{K})$, $R=R^{\delta}, S=S^{\delta}$, where $X^{\delta}$ stands for the symmetric of $X$ by the antidiagonal. A Borel subgroup of $S p(V, \omega)$ is formed by the upper triangular matrices $g$ such that $\left(g^{\mathrm{t}}\right) K g=K$, i.e., the blockwise matrices of the form $g=\left(\begin{array}{cc}P & P R \\ 0 & \left(P^{-1}\right)^{\delta}\end{array}\right)$ with $P \in G L_{p}(\mathbb{K})$ upper triangular and $R \in M_{p}(\mathbb{K})$ such that $R=R^{\delta}$.

A nilpotent element $e \in \mathfrak{s p}(V, \omega)$ is an antiadjoint endomorphism, which is nilpotent in the usual sense. Its Jordan form $\pi=\left(\pi_{1} \geq \ldots \geq \pi_{k}\right)$ is a partition of $n$ where, for every odd integer $q$, the number of parts $\pi_{i}$ that are equal to $q$ is even. Conversely, if $\pi$ is a partition satisfying this property, then it occurs as the Jordan form of a nilpotent element of $\mathfrak{s p}(V, \omega)$, which is uniquely determined up to its nilpotent orbit. As in Section 6.1 we represent $\pi$ by a Young diagram that we view as a set of boxes, and we view $\pi_{i}$ as the subset of boxes in the $i$-th row of $\pi$. In fact, we partition the set of rows of $\pi$ by writing

$$
\pi=\left(\pi_{1}^{+} \sqcup \pi_{1}^{-}\right) \sqcup \ldots \sqcup\left(\pi_{\ell}^{+} \sqcup \pi_{\ell}^{-}\right) \sqcup\left(\pi_{1}^{0} \sqcup \ldots \sqcup \pi_{m}^{0}\right)
$$

where:

- $\left\{\pi_{1}^{+}, \pi_{1}^{-}\right\}, \ldots,\left\{\pi_{\ell}^{+}, \pi_{\ell}^{-}\right\}$are $\ell$ pairwise distinct pairs of rows such that, for each $i, \pi_{i}^{+}, \pi_{i}^{-}$have the same length, with $\ell$ maximal;

- $\pi_{1}^{0}, \ldots, \pi_{m}^{0}$ are the remaining rows, whose lengths are therefore pairwise distinct, even.

Thus $m$ is the number of parts of the partition $\pi$, which occur with odd multiplicity. The following fact is shown in [10, Theorem A.3].

Proposition 8. The nilpotent element $e \in \mathfrak{s p}(V, \omega)$ is of standard Levi type if and only if $m \in\{0,1\}$.

For the moment, we do not assume that $e$ is of standard Levi type (hence $m$ is arbitrary). To each box $a \in \pi$, we attach a sign $\varepsilon(a) \in\{1,-1\}$ and a dual box $a^{*} \in \pi$ in the following way:

- if $a, a^{\prime}$ are, respectively, the $j$-th box from the left in $\pi_{i}^{+}$and the $j$-th box from the right in $\pi_{i}^{-}$, then set $\varepsilon(a)=(-1)^{j}=-\varepsilon\left(a^{\prime}\right), a^{*}=a^{\prime}$, and $a^{\prime *}=a$;

- if $a, a^{\prime}$ are, respectively, the $j$-th box from the left and the $j$-th box from the right in $\pi_{i}^{0}$, then set $\varepsilon(a)=(-1)^{j}$ and $a^{*}=a^{\prime}$. 
There is a basis $\left\{v_{a}: a \in \pi\right\}$ parametrized by the boxes of $\pi$ and satisfying the following requirements:

- $e\left(v_{a}\right)=0$ if $a$ belongs to the first column of $\pi$;

- $e\left(v_{a}\right)=v_{a^{\prime}}$ where $a^{\prime}$ denotes the box next to $a$ on the left, otherwise;

- $\omega\left(v_{a}, v_{a^{\prime}}\right)=\varepsilon(a)$ if $a^{\prime}=a^{*}$, and $\omega\left(v_{a}, v_{a^{\prime}}\right)=0$ otherwise.

Thus $\left\{v_{a}\right\}$ is a Jordan basis for the endomorphism $e$, where the Jordan blocks $V_{i}^{\epsilon}:=\left\langle v_{a}: a \in \pi_{i}^{\epsilon}\right\rangle_{\mathbb{K}}$ correspond to the rows $\pi_{i}^{\epsilon}$ of $\pi$, for all $i$, all $\epsilon \in\{+, 0,-\}$.

Given $\left(t_{1}, \ldots, t_{\ell}\right) \in\left(\mathbb{K}^{*}\right)^{\ell}$, let $h\left(t_{1}, \ldots, t_{\ell}\right) \in S p(V, \omega)$ be given by $v_{a} \mapsto t_{i} v_{a}$ for $a \in \pi_{i}^{+}, v_{a} \mapsto t_{i}^{-1} v_{a}$ for $a \in \pi_{i}^{-}$, and $v_{a} \mapsto v_{a}$ for all $a \in \pi_{i}^{0}$. Then, $S_{0}:=$ $\left\{h\left(t_{1}, \ldots, t_{\ell}\right)\right\} \cong\left(\mathbb{K}^{*}\right)^{\ell}$ is a maximal torus of $Z_{G}(e)$.

A cocharacter associated to $e$ is obtained as follows. For every $a \in \pi_{i}^{\epsilon}$ we set

$$
\nu(a)=\#\left\{\text { boxes of } \pi_{i}^{\epsilon} \text { on the right of } a\right\}-\#\left\{\text { boxes of } \pi_{i}^{\epsilon} \text { on the left of } a\right\}
$$

for all $i$, all $\epsilon \in\{+, 0,-\}$. Then, let $\tau: \mathbb{K}^{*} \rightarrow S p(V, \omega)$ be defined by

$$
\tau(t): v_{a} \mapsto t^{\nu(a)} v_{a} \text { for all } a \in \pi .
$$

It is easy to see that $\tau$ is a cocharacter associated to $e$. It is also straightforward to check that the torus $S_{0} \tau\left(\mathbb{K}^{*}\right)$ is regular if and only if $m \in\{0,1\}$, thus one can retrieve Proposition 8 by applying Proposition 1 .

Finally (without necessarily assuming that $e$ is of standard Levi type), we construct a Borel subgroup adapted to $\left(S_{0}, \tau\right)$. Consider the order $\prec$ on the set of rows $\left\{\pi_{1}, \ldots, \pi_{k}\right\}=\left\{\pi_{i}^{+}, \pi_{j}^{-}, \pi_{h}^{0}\right\}$ given by

$$
\pi_{1}^{+} \prec \ldots \prec \pi_{\ell}^{+} \prec \pi_{1}^{0} \prec \ldots \prec \pi_{m}^{0} \prec \pi_{\ell}^{-} \prec \ldots \prec \pi_{1}^{-} .
$$

There is a unique total order $<$ on $\pi$ such that:

- $\nu(a) \geq \nu\left(a^{\prime}\right)$ whenever $a<a^{\prime}$;

- if $a \in \pi_{i}$ and $a^{\prime} \in \pi_{j}$ satisfy $\nu(a)=\nu\left(a^{\prime}\right)$, then $a<a^{\prime} \Leftrightarrow \pi_{i} \prec \pi_{j}$.

Let $a_{1}<\ldots<a_{n}$ be the boxes of $\pi$ written in increasing order. It is easy to check that $a_{i}^{*}=a_{n-i+1}$ for all $i \in\{1, \ldots, n\}$. Setting $v_{i}=\varepsilon\left(a_{i}\right) v_{a_{i}}$ for $i \in\{1, \ldots, p\}$, and $v_{i}=v_{a_{i}}$ for $i \in\{p+1, \ldots, 2 p\}$, we get a basis satisfying (18). The subgroup $B \subset S p(V, \omega)$ of automorphisms that are upper triangular in the basis $\left(v_{1}, \ldots, v_{n}\right)$ is a Borel subgroup (see Remark 4), which is adapted to $\left(S_{0}, \tau\right)$.

Example 4. Assume $V=\mathbb{K}^{10}$ and let $e \in \mathfrak{s p}(V, \omega)$ be a nilpotent element of Jordan form $\pi=(3,3,2,1,1)$, represented by the Young diagram:

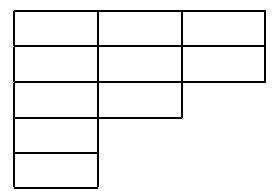

By Proposition 8, $e$ is of standard Levi type. We denote by $\pi_{1}^{+}, \pi_{2}^{+}$the first and fourth rows of $\pi$, by $\pi_{1}^{-}, \pi_{2}^{-}$the second and fifth rows, and by $\pi_{1}^{0}$ the third row. Putting the weight $\nu(a)$ in each box $a$, we get:

\begin{tabular}{|c|c|c|}
\hline 2 & 0 & -2 \\
\hline 2 & 0 & -2 \\
\hline 1 & -1 & \multicolumn{2}{|c}{} \\
\cline { 1 - 1 } 0 & \multicolumn{2}{|c}{} \\
\cline { 1 - 1 } 0 & \multicolumn{2}{|c}{}
\end{tabular}


The order on the boxes $a_{1}, \ldots, a_{10}$ of the diagram is indicated in the following tableau, where we put $i$ in the box $a_{i}$ :

\begin{tabular}{|c|c|c|}
\hline 1 & 4 & 9 \\
\hline 2 & 7 & 10 \\
\hline 3 & 8 & \multicolumn{1}{|c}{} \\
\cline { 1 - 1 } 5 & \multicolumn{2}{|c}{} \\
\cline { 1 - 1 } 6 & \multicolumn{2}{|c}{} \\
\cline { 1 - 2 } &
\end{tabular}

We get

$$
v_{i}=v_{a_{i}} \text { for } i \in\{2,4,6,7,8,9,10\} \text { and } v_{i}=-v_{a_{i}} \text { for } i \in\{1,3,5\} .
$$

The matrix of $e$ in the basis $\left(v_{1}, \ldots, v_{10}\right)$ is then the following:

$$
\left(\begin{array}{cccccccccc}
0 & 0 & 0 & -1 & 0 & 0 & 0 & 0 & 0 & 0 \\
0 & 0 & 0 & 0 & 0 & 0 & 1 & 0 & 0 & 0 \\
0 & 0 & 0 & 0 & 0 & 0 & 0 & -1 & 0 & 0 \\
0 & 0 & 0 & 0 & 0 & 0 & 0 & 0 & 1 & 0 \\
0 & 0 & 0 & 0 & 0 & 0 & 0 & 0 & 0 & 0 \\
0 & 0 & 0 & 0 & 0 & 0 & 0 & 0 & 0 & 0 \\
0 & 0 & 0 & 0 & 0 & 0 & 0 & 0 & 0 & 1 \\
0 & 0 & 0 & 0 & 0 & 0 & 0 & 0 & 0 & 0 \\
0 & 0 & 0 & 0 & 0 & 0 & 0 & 0 & 0 & 0 \\
0 & 0 & 0 & 0 & 0 & 0 & 0 & 0 & 0 & 0
\end{array}\right)
$$

The tori $S_{0}$ and $\tau\left(\mathbb{K}^{*}\right)$ correspond to the subgroups of matrices of the forms

$$
\Delta\left(t_{1}, t_{1}^{-1}, 1, t_{1}, t_{2}, t_{2}^{-1}, t_{1}^{-1}, 1, t_{1}, t_{1}^{-1}\right) \text { and } \Delta\left(t^{2}, t^{2}, t, 1,1,1,1, t^{-1}, t^{-2}, t^{-2}\right),
$$

where $\Delta\left(t_{1}, \ldots, t_{n}\right)$ stands for the $n \times n$ sized diagonal matrix with entries $t_{1}, \ldots, t_{n}$ along the diagonal. Finally, $B \subset S p(V, \omega)$ corresponds to the group of upper triangular matrices $g \in S L_{10}(\mathbb{K})$ described in Remark 4 .

Remark 5. The case of the orthogonal group is similar. Let $V$ be a vector space of finite dimension $n \geq 1$ (even or odd), endowed with an orthogonal form $\phi$ : $V \times V \rightarrow \mathbb{K}$. Let $G=S O(V, \phi) \subset S L(V)$ be the subgroup of automorphisms that preserve $\phi$, its Lie algebra is $\mathfrak{g}=\mathfrak{s o}(V, \phi)$ the space of endomorphisms of trace 0 , which are antiadjoint with respect to $\phi$. A nilpotent element $e \in \mathfrak{s o}(V, \phi)$ is an antiadjoint endomorphism, which is nilpotent in the usual sense. Its Jordan form $\pi=\left(\pi_{1} \geq \ldots \geq \pi_{k}\right)$ is a partition of $n$ where the even parts occur with even multiplicities. Let $\pi_{1}^{0}, \ldots, \pi_{m}^{0} \in\left\{\pi_{1}, \ldots, \pi_{k}\right\}$ be the values occurring with odd multiplicities in the partition $\pi$. It is shown in [10, Theorems A.2, A.4] that $e$ is of standard Levi type if and only if

$$
m \in\{0,1\} \text { or }\left(m=2 \text { and } 1 \in\left\{\pi_{1}^{0}, \pi_{2}^{0}\right\}\right) .
$$

This conclusion can be retrieved through Proposition 1, In fact, following the same scheme as above, one can construct a maximal torus $S_{0} \subset Z_{G}(e)$, a cocharacter $\tau$ associated to $e$, and a Borel subgroup adapted to $\left(S_{0}, \tau\right)$, to which the results of this paper can be applied.

\section{ACKNOWLEDGEMENT}

The author is grateful to the referee for valuable comments. 


\section{REFERENCES}

[1] A. Białynicki-Birula, Some theorems on actions of algebraic groups, Ann. of Math. (2) 98 (1973), 480-497. MR0366940 (51 \#3186)

[2] Walter Borho and Hanspeter Kraft, Uber Bahnen und deren Deformationen bei linearen Aktionen reduktiver Gruppen (German, with English summary), Comment. Math. Helv. 54 (1979), no. 1, 61-104, DOI 10.1007/BF02566256. MR522032 (82m:14027)

[3] Walter Borho and Robert MacPherson, Partial resolutions of nilpotent varieties, Analysis and topology on singular spaces, II, III (Luminy, 1981), Astérisque, vol. 101, Soc. Math. France, Paris, 1983, pp. 23-74. MR737927 (85j:14087)

[4] Jonathan Brundan and Victor Ostrik, Cohomology of Spaltenstein varieties, Transform. Groups 16 (2011), no. 3, 619-648, DOI 10.1007/s00031-011-9149-2. MR2827037

[5] Roger W. Carter, Finite groups of Lie type, Pure and Applied Mathematics (New York), John Wiley \& Sons, Inc., New York, 1985. Conjugacy classes and complex characters; A Wiley-Interscience Publication. MR794307 (87d:20060)

[6] David H. Collingwood and William M. McGovern, Nilpotent orbits in semisimple Lie algebras, Van Nostrand Reinhold Mathematics Series, Van Nostrand Reinhold Co., New York, 1993. MR $1251060(94 \mathrm{j}: 17001)$

[7] C. De Concini, G. Lusztig, and C. Procesi, Homology of the zero-set of a nilpotent vector field on a flag manifold, J. Amer. Math. Soc. 1 (1988), no. 1, 15-34, DOI 10.2307/1990965. MR924700 (89f:14052)

[8] F. De Mari, C. Procesi, and M. A. Shayman, Hessenberg varieties, Trans. Amer. Math. Soc. 332 (1992), no. 2, 529-534, DOI 10.2307/2154181. MR1043857 (92j:14060)

[9] Lucas Fresse and Anna Melnikov, Some characterizations of singular components of Springer fibers in the two-column case, Algebr. Represent. Theory 14 (2011), no. 6, 1063-1086, DOI 10.1007/s10468-010-9227-5. MR2844756 (2012m:14087)

[10] Lucas Fresse and Anna Melnikov, Smooth orbital varieties and orbital varieties with a dense B-orbit, Int. Math. Res. Not. IMRN 5 (2013), 1122-1203. MR3031828

[11] Jens Carsten Jantzen, Nilpotent orbits in representation theory, Lie theory, Progr. Math., vol. 228, Birkhäuser Boston, Boston, MA, 2004, pp. 1-211. MR2042689 (2005c:14055)

[12] Daniel Juteau, Carl Mautner, and Geordie Williamson, Parity sheaves, J. Amer. Math. Soc. 27 (2014), no. 4, 1169-1212, DOI 10.1090/S0894-0347-2014-00804-3. MR3230821

[13] Hiraku Nakajima, Homology of moduli spaces of instantons on ALE spaces. I, J. Differential Geom. 40 (1994), no. 1, 105-127. MR.1285530 (95g:58031)

[14] D. I. Panyushev, Rationality of singularities and the Gorenstein property of nilpotent orbits (Russian), Funktsional. Anal. i Prilozhen. 25 (1991), no. 3, 76-78, DOI 10.1007/BF01085494; English transl., Funct. Anal. Appl. 25 (1991), no. 3, 225-226 (1992). MR1139878(92i:14047)

[15] Martha Precup, Affine pavings of Hessenberg varieties for semisimple groups, Selecta Math. (N.S.) 19 (2013), no. 4, 903-922, DOI 10.1007/s00029-012-0109-z. MR3131491

[16] Naohisa Shimomura, The fixed point subvarieties of unipotent transformations on the flag varieties, J. Math. Soc. Japan 37 (1985), no. 3, 537-556, DOI 10.2969/jmsj/03730537. MR792991 (87a:14041)

[17] N. Spaltenstein, On unipotent and nilpotent elements of groups of type $E_{6}$, J. London Math. Soc. (2) 27 (1983), no. 3, 413-420, DOI 10.1112/jlms/s2-27.3.413. MR697134(84h:20034)

[18] Nicolas Spaltenstein, Classes unipotentes et sous-groupes de Borel (French), Lecture Notes in Mathematics, vol. 946, Springer-Verlag, Berlin-New York, 1982. MR672610 (84a:14024)

[19] T. A. Springer, Linear algebraic groups, 2nd ed., Progress in Mathematics, vol. 9, Birkhäuser Boston, Inc., Boston, MA, 1998. MR1642713 (99h:20075)

[20] Robert Steinberg, On the desingularization of the unipotent variety, Invent. Math. 36 (1976), 209-224. MR0430094 (55 \#3101)

[21] Julianna S. Tymoczko, Linear conditions imposed on flag varieties, Amer. J. Math. 128 (2006), no. 6, 1587-1604. MR2275912(2007h:14070)

[22] Nanhua Xi, A partition of the Springer fibers $\mathcal{B}_{N}$ for type $A_{n-1}, B_{2}, G_{2}$ and some applications, Indag. Math. (N.S.) 10 (1999), no. 2, 307-320, DOI 10.1016/S0019-3577(99)80024-X. MR:1816223(2001m:20075)

Université de Lorraine, CNRS, Institut Élie Cartan de Lorraine, UMR 7502, VanDOEUVRE-LÈS-NANCY, F-54506, FrANCE

E-mail address: lucas.fresse@univ-lorraine.fr 